\title{
Parameters of rotating neutron stars with and without hyperons (Research Note)
}

\author{
M. Bejger \\ N. Copernicus Astronomical Center, Bartycka 18, 00-716 Warsaw, Poland \\ e-mail: bejger@camk.edu.pl \\ Received 10 December 2012 / Accepted 15 February 2013 \\ ABSTRACT

\begin{abstract}
Context. The discovery of a $2 M_{\odot}$ neutron star provided a robust constraint for the theory of exotic dense matter, bringing into question the existence of strange baryons in the interiors of neutron stars. Although many theories fail to reproduce this observational result, several equations of state containing hyperons are consistent with it.

Aims. We study global properties of stars using equations of state containing hyperons, and compare them to those without hyperons to find similarities, differences, and limits that can be compared with the astrophysical observations.

Methods. Rotating, axisymmetric, and stationary stellar configurations in general relativity are obtained, and their global parameters are studied.

Results. Approximate formulæ describing the behavior of the maximum and minimum stellar mass, compactness, surface redshifts, and moments of inertia as functions of spin frequency are provided. We also study the thin disk accretion and compare the spin-up evolution of stars with different moments of inertia.
\end{abstract}

Key words. stars: neutron - equation of state - stars: rotation - dense matter

\section{Introduction}

The discovery of a $2 M_{\odot}$ neutron star (NS; Demorest et al. 2010) provides a strong motivation for (the community of) densematter physicists to understand the role of exotic phases in the interior of neutron stars. The appearance of new particles (in general, new phases of matter) typically results in the softening of matter, since the contribution to pressure from energetic nucleons is replaced by that of slowly-moving, massive particles; the pressure increases less steeply with the density in the equation of state (EOS), which in turn lowers the NS maximum allowable mass (for a review concerning dense matter EOSs, see Haensel et al. 2007). The state of the dense matter in the NS cores is very different from the state known at the energies and densities reached by the terrestrial experiments; notably, stable strange matter may appear in the interiors of NSs at densities a few times the nuclear saturation density, with strange baryons (hyperons) being a very probable result.

We study a set of selected EOSs, representing current theoretical approaches to the description of the dense matter, that are consistent with the robust constraint put forward by the observers. If we assume that hyperons do exist in the interiors of massive NSs, then based on our present knowledge it seems necessary for such objects to be composed of sufficiently stiff matter (at lower densities) to sustain the softening that is introduced by the appearance of hyperons. In other words, substantial stiffness of the EOS of nucleonic (non-strange) matter is a necessary condition for the existence of hyperons in the core of a massive NS. Consequently, stellar configurations that contain hyperons will, at least partly, differ from those without hyperons. We therefore seek similarities and differences between them, as well as limits that can be applied to astrophysical observations.
This Note is composed as follows: Sect. 2 contains the description of methods and EOSs used; Sect. 3 presents the results for the gravitational mass, surface redshift, moment of inertia and accretion tracks; Sect. 4 contains the conclusions and summary.

\section{Equations of state}

The following selection of nucleonic EOSs is used: the APR EOS (Akmal et al. 1998, model $A 18+\delta v+U I X^{\star}$ ) is a variational, non-relativistic many-body solution with relativistic corrections; the DH EOS (Douchin \& Haensel 2001) is constructed using a non-relativistic energy density functional based on the SLy4 effective nuclear interaction, designed to describe both crust and core in a unified way; the BSK20 EOS (moderate stiffness EOS by Goriely et al. 2010) is also based on the nuclear energy-density functional theory, using the generalized Skyrme forces fitted to experimental nuclear data and reproducing properties of infinite nuclear matter from the calculations of many-body interactions.

Realistic microscopic dense-matter theories (the BruecknerHartree-Fock approach) predict maximum masses of NSs with hyperons much below the observed $\simeq 2 M_{\odot}$ (see e.g., Schulze et al. 2006; Burgio et al. 2011, and references therein). This may be caused by a limited knowledge of hyperon-hyperon and hyperon-nucleon three-body forces; see, however, a recent investigation of Vidaña et al. (2011), who estimated the effect of three-body forces in the hyperonic sector on the NS maximum mass. Assuming that hyperon interactions are weaker than the pure nucleonic interactions, the resulting non-rotating NS maximum mass is located in the $1.27-1.6 M_{\odot}$ range. The 
Table 1. Selected parameters of static configurations.

\begin{tabular}{lcccccc}
\hline \hline EOS & $M_{\max }^{\mathrm{s}}$ & $R\left(M_{\max }^{\mathrm{s}}\right)$ & $I_{\max }^{\mathrm{s}}$ & $R_{1.4}$ & $I_{1.4}$ & $f^{\mathrm{s}}$ \\
\hline APR & 2.19 & 9.93 & 2.24 & 11.34 & 1.31 & 2740.47 \\
BSK20 & 2.17 & 10.17 & 2.00 & 11.75 & 1.39 & 2630.50 \\
DH & 2.05 & 9.99 & 2.27 & 11.73 & 1.37 & 2630.63 \\
BM165 & 2.03 & 10.68 & 2.24 & 13.59 & 1.74 & 2367.82 \\
DS08 & 2.05 & 12.02 & 2.56 & 13.91 & 1.81 & 1993.42 \\
GM1Z0 & 2.29 & 12.00 & 3.09 & 13.89 & 1.84 & 2108.51 \\
TM1C & 2.05 & 12.51 & 2.72 & 14.51 & 1.91 & 1873.41 \\
\hline
\end{tabular}

Notes. Mass expressed in $M_{\odot}$, radius $R$ in $\mathrm{km}$, the moment of inertia $I$ in $10^{45} \mathrm{~g} \mathrm{~cm}^{2}$, and the frequency $f^{\mathrm{s}}=(1 / 2 \pi) \sqrt{G M_{\max }^{\mathrm{s}} / R^{3}\left(M_{\max }^{\mathrm{s}}\right)}$ in $\mathrm{Hz}$; $R_{1.4}$ and $I_{1.4}$ are computed for $M=1.4 M_{\odot}$.

problematic feature of low $M_{\max }$ is usually remedied by providing stronger repulsion between the hyperons and/or letting them appear only at very high densities. Below, we list a selection of EOSs with hyperons, derived from theories that successfully deal with the problem of a massive NS: the DS08 EOS (Dexheimer \& Schramm 2008) uses an effective hadronic SU(3) chiral model including the baryon octet and fourth-order selfinteraction terms of the $\omega, \rho$, and $\phi$ vector mesons; the GM1Z0 EOS (Weissenborn et al. 2012) is based on a relativistic mean field model which allows the study of the departure from a vector meson-hyperon couplings stemming from the SU(6) quark model to a more general SU(3) prescription; the GM1 model with the ratio between the meson octet and the singlet coupling constant $z=g_{8} / g_{1}=0$ (all the baryon-meson couplings are equal) was used, which makes it the stiffest in our sample. The BM165 EOS (Bednarek et al. 2011) was also derived from a relativistic mean field model, with a non-linear Lagrangian that includes quatric terms in the meson fields, and two additional hidden-strangeness mesons $\sigma^{*}$ and $\phi$ that couple to hyperons only. The TM1C EOS (Gusakov et al., priv. comm.) employs a prescription similar to the BM165 EOS for purely nucleonic matter, and also introduces scalar $\sigma^{*}$ and vector $\phi$ mesons in the description of hyperon interaction, with an additional $\Lambda-\Lambda$ hyperon attraction. The SU(6) symmetry breaking applied in the model amounts to $z=0.2$.

\section{Results}

The following subsections contain the results for constant spin frequency tracks (sequences of configurations) of rotating stars. The frequency range spans an astrophysically-relevant range from $f=0 \mathrm{~Hz}$ (static configurations, see Table 1 for their representative parameters) up to $f=1200 \mathrm{~Hz}$ (i.e., much above the frequency of $716 \mathrm{~Hz}$ of the most rapid pulsar to date, PSR J17482446ad of Hessels et al. 2006). Rigidly-rotating, stationary, and axisymmetric stellar configurations were obtained by means of the numerical library LORENE ${ }^{1}$, nrotstar code, using the formulation of Bonazzola et al. (1993), with the accuracy checked by a 2D virial theorem (Bonazzola \& Gourgoulhon 1994). The sequences are limited by the following conditions: from the low central density end, they terminate at the so-called massshedding limit, when the orbital frequency of a test particle at the star's equator equals the stellar spin frequency $f$. The high central density limit is marked by the onset of the axisymmetric perturbation instability described by the condition $\partial M /\left.\partial \lambda\right|_{J}=0$, where $M$ is the gravitational mass and $\lambda$ a suitable parametrization of the sequence of configurations (e.g., the central density;

\footnotetext{
1 http://www. lorene.obspm.fr
}

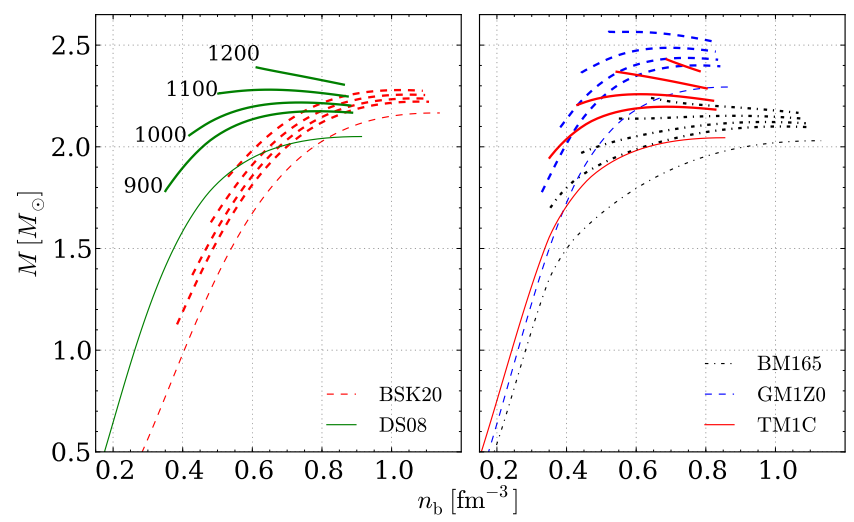

Fig. 1. Gravitational mass-central baryon density $M-n_{\mathrm{b}}$ relations for selected spin frequencies $f$, for stars composed of the BSK20 EOS (dashed red lines) and four hyperonic EOSs: DS08 (solid green), BM165 (dash-dotted black), GM1Z0 EOS (dashed blue) and TM1C (solid red lines). High- and low-density ends correspond to the axisymmetric instability and the mass-shedding limits, respectively. From bottom to top for each EOS, $f$ equals 0, 900, 1000, 1100 and $1200 \mathrm{~Hz}$. (Color online.)

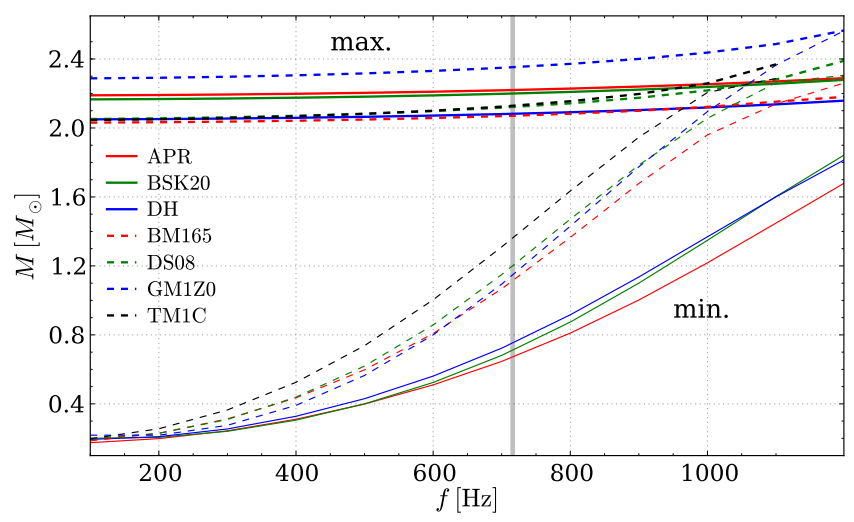

Fig. 2. Range of allowable gravitational masses as a function of spin frequency $f$. The vertical line indicates $716 \mathrm{~Hz}$ frequency (spin frequency of the most-rapidly rotating NS to date). (Color online.)

see Friedman et al. 1988 for details). We define the moment of inertia as $I=J / \Omega$, where $J$ is the total stellar angular momentum and $\Omega$ is the angular frequency, $\Omega=2 \pi f$ (for the definitions of $M$ and $J$, see Bonazzola et al. 1993). For non-rotating configurations, $I$ is calculated using the slow-rotation approximation (Hartle 1967).

Parameters of the approximate formulæ presented below were obtained by the $\chi^{2}$ fitting, yielding a typical accuracy of the order of one per cent.

\subsection{Gravitational mass}

Figure 1 shows how the mass-central baryon density $M\left(n_{\mathrm{b}}\right)$ relation changes with $f$ for the selected EOSs. A strong reduction of the available mass and the central density range for a large $f$ is characteristic of hyperonic EOSs; moreover, configurations near the mass-shedding limit (left sides of curves) may be more massive than those near the axisymmetric perturbation instability limit (right sides). Figure 2 shows the range of available masses as a function of spin frequency $f$; we note that the mass of a hyperonic EOS star is already strongly constrained by the existence of $716 \mathrm{~Hz}$ pulsar (Hessels et al. 2006). Detection of a $f \simeq 800 \mathrm{~Hz}$ pulsar would mean that such a stiff EOS cannot 

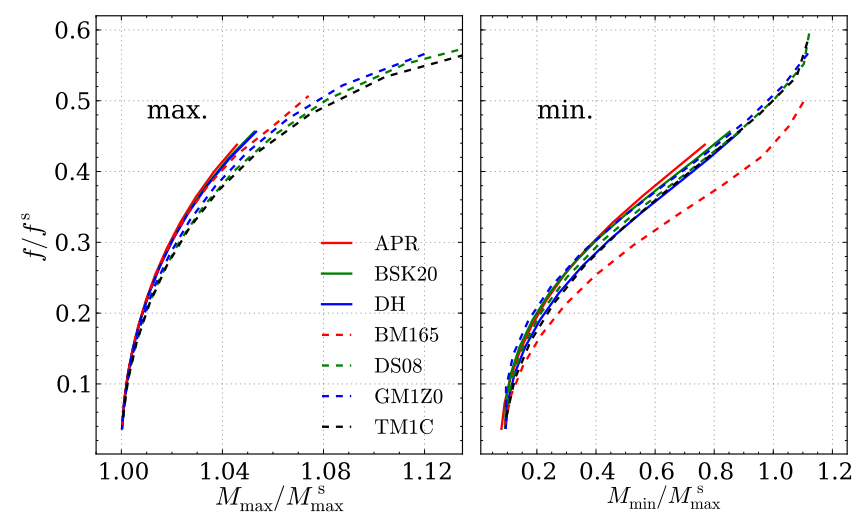

Fig. 3. Maximum mass $M_{\max }$ (left panel) and minimum mass $M_{\min }$ (right panel) of rotating NSs as a function of the spin frequency $f$. Values are scaled by the parameters of non-rotating configurations. (Color online.)

yield a star with $M<1.4 M_{\odot}$. (In the case of the TM1C EOS this minimum mass is already approached for $716 \mathrm{~Hz}$ ). The growth of the maximum mass $M_{\max }$, normalized by the maximum mass of the static configuration $M_{\max }^{\mathrm{s}}$ can be approximated by

$M_{\max } / M_{\max }^{\mathrm{s}}=0.49\left(f / f^{\mathrm{s}}\right)^{8 / 3}+1$,

where $f^{\mathrm{s}}=(1 / 2 \pi) \sqrt{G M_{\max }^{\mathrm{s}} / R^{3}\left(M_{\mathrm{max}}^{\mathrm{s}}\right)}$ is the frequency scaling factor $^{2}$ formed of a static star maximum-mass parameters, gravitational mass $M_{\max }^{\mathrm{s}}$ and corresponding radius $R\left(M_{\max }^{\mathrm{s}}\right)$. In the case of minimum mass $M_{\text {min }}$, one can obtain a similar (although somewhat cruder) approximation to the one for the $M_{\max }$,

$M_{\min } / M_{\max }^{\mathrm{s}}=3.57\left(f / f^{\mathrm{s}}\right)^{2}+0.1$.

The above relation underestimates the $M_{\min } / M_{\max }^{\mathrm{s}}$ for BM165 EOS stars for a given frequency, because the maximum-mass radius $R\left(M_{\max }\right)$ is smaller than for other hyperonic EOSs, resulting in a larger $f^{\mathrm{s}}$.

\subsection{Surface redshifts}

Surface redshift may provide important information about the spacetime in the vicinity of the NS. In the case of a rotating star, one considers the redshift $z_{\mathrm{p}}$ of photons coming from the pole, as well as two equatorial redshifts for photons, emitted tangentially in and opposite the direction of rotation (forward $z_{\mathrm{f}}$ and backward $z_{\mathrm{b}}$ redshift, respectively; for definitions, see e.g., Gourgoulhon 2010, Sect. 4.6). The left panel in Fig. 4 shows the relations for $z$ for the minimum compactness $\left(M / R_{\text {eq }}\right)_{\min }$, i.e., configurations at the mass-shedding limit, that bear some resemblance to the configurations during the photospheric radius expansion burst (Steiner et al. 2010 and references therein); $R_{\mathrm{eq}}$ denotes the equatorial radius. These relations may be regarded as upper limits on the compactness parameter for a measured $z$. We approximate $z_{\mathrm{p}}$ and $z_{\mathrm{b}}$ by $a(M / R)^{3 / 2}+b$, where $a=9.62$ and $b=0.023$ for $z_{\mathrm{p}}$, and $a=25.11$ and $b=0.18$ for $z_{\mathrm{b}} ; z_{\mathrm{f}}$ is approximated by $-0.68(M / R)^{0.4}$.

The right panel shows redshift functions for the maximumcompactness $\left(M / R_{\text {eq }}\right)_{\max }$ configuration along the line corresponding to the axisymmetric perturbation instability limit. For spin frequencies under consideration, it may be also treated as an approximate lower limit on the stellar mass. We propose

$2 f^{\mathrm{s}}$ equals the orbital frequency of a test particle orbiting spherical mass $M_{\max }^{\mathrm{s}}$ at a distance $R\left(M_{\max }^{\mathrm{s}}\right)$.

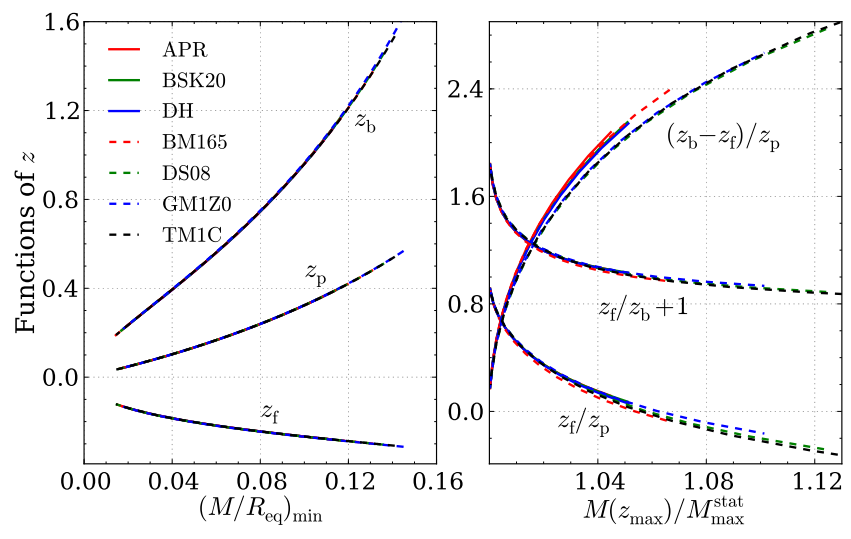

Fig. 4. Surface redshifts for rotating configurations. Left panel: equatorial backward (top curve) and forward (bottom curve) redshifts $z_{\mathrm{b}}$ and $z_{\mathrm{f}}$, and the polar redshift $z_{\mathrm{p}}$ for configurations with the minimal mass, as functions of the compactness $\left(M / R_{\mathrm{eq}}\right)_{\min }$. Right panel: functions of the three redshifts for rotating, maximally-compact configurations. (Color online.)

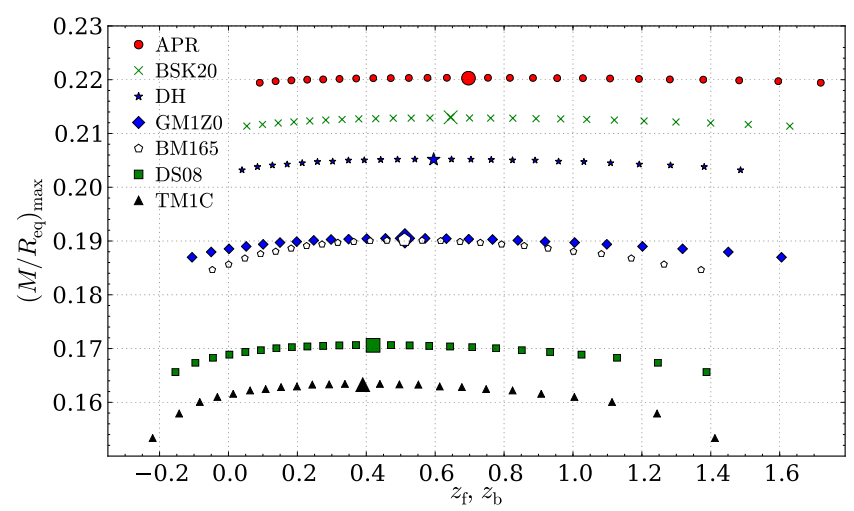

Fig. 5. Maximal compactness vs. equatorial redshifts $z_{\mathrm{f}}$ and $z_{\mathrm{b}}$ for different rotation rates; for $z_{\mathrm{f}}, f$ increases to the left of the bigger symbol that denotes the static configuration; for $z_{\mathrm{b}}, f$ increases to the right. Points correspond to frequencies from 0 to $1200 \mathrm{~Hz}$. (Color online.)

parametrizing these relations as follows: for $\zeta=\left(z_{\mathrm{b}}-z_{\mathrm{f}}\right) / z_{\mathrm{p}}$, $M / M_{\max }^{\mathrm{s}} \simeq 0.008 \zeta^{2.5}+1$; for $\zeta=1-z_{\mathrm{f}} / z_{\mathrm{p}}, M / M_{\max }^{\mathrm{s}}$ is approximated by $0.06 \zeta^{2.5}+1$. In the case of the third function, we chose $\zeta=1-z_{\mathrm{f}} / z_{\mathrm{b}}$, and $M / M_{\max }^{\mathrm{s}} \simeq 0.04 \zeta^{6}+0.02 \zeta^{3}+1$. The value of $z_{\mathrm{b}}-z_{\mathrm{f}}$ quantifies the redshift effect on the maximal line broadening coming from the equator of a rotating star. Figure 5 presents the maximal compactness for such stars against the two equatorial redshifts. The $\left(M / R_{\mathrm{eq}}\right)_{\max }$ stays remarkably constant with rotation, and could be used to distinguish between the two sets of models.

\subsection{Moment of inertia}

A potentially interesting NS parameter is the moment of inertia $I$ (in principle measurable using the spin-orbit coupling, Lattimer $\&$ Schutz 2005). Figure 6 shows the behavior of the gravitational mass $M$ as a function of the moment of inertia $I$ for static configurations. The curves in the left panel may be approximated by a straight line, $I(M)=a_{\mathrm{I}} M+b_{\mathrm{I}}$. For nucleonic EOSs, $a_{\mathrm{I}}=1.22$ and $b_{\mathrm{I}}=-0.34$, for hyperonic EOSs $a_{\mathrm{I}}=1.51$. In the right panel we plot the value of $I / R^{6}$ as a function of $M$; this value enters the estimation of the minimum magnetic dipole field at the pulsar surface, $B>\left(I / R^{6}\right)^{1 / 2}\left(3 c^{3} \dot{P} P / 8 \pi^{2}\right)^{1 / 2}$, where $P=2 \pi / f$ 

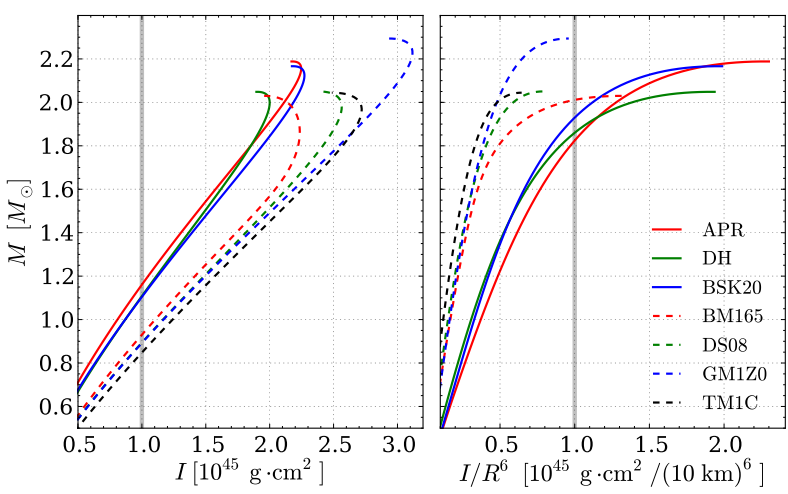

Fig. 6. Left panel: moment of inertia $I$ as a function of gravitational mass $M$ for static solutions. Right panel: moment of inertia $I$ divided by $R^{6}$ (as used in the estimation of the minimum surface magnetic field of a pulsar) for static stars. Vertical lines correspond to a fiducial model of $I=10^{45} \mathrm{~g} \mathrm{~cm}^{2}$ and $10 \mathrm{~km}$. (Color online.)

denotes the spin period ${ }^{3}$. Figure 7 presents how the available range of the moments of inertia changes with rotation. For the frequencies $f \geq 800 \mathrm{~Hz}$ the moment of inertia tend to be already quite constrained. The minimum value of $I$ for sub-millisecond rotation coincides with the high-density end of the track, while the mass-shedding limit is characterized by a large $I$ (because of a strong equatorial radius dependence, the effect is much more pronounced than for the gravitational mass, Fig. 2). As many softer EOSs are now ruled out by observations, we reproduce the formula suggested by Lattimer \& Prakash (2001) in the form of Bejger \& Haensel (2002), with slightly different coefficients in the static case. For an astrophysically relevant range of masses $M>0.5 M_{\odot}$ we have

$I / M R^{2}=a_{0}(M / R)+b_{0}$,

with $a_{0}=1.40$ and $b_{0}=0.19$ (left panel in Fig. 8). This approximation is generally valid for the static results in our sample. A more universal formula, which takes rotation into account, will depend on the spin frequency $f$; the compactness $M / R$ must be re-defined as the gravitational mass $M$ to the circumferential radius $R_{\text {eq }}$ ratio. For EOSs without hyperons, we propose the following approximation:

$a(f)=0.19 f_{1}^{4}+a_{0} \quad$ and $\quad b(f)=-0.042 f_{1}^{4}+b_{0}$,

where $f_{1}=f /(1 \mathrm{kHz})$. Since the rotation influences hyperonic stars in a different way, we produce an analogous approximation for a subset of hyperonic EOSs only. In the static case we obtain $a_{0 h}=1.14$ and $b_{0 h}=0.22$, and for rotating configurations $a(f)=0.51 f_{1}^{4}+a_{0 h} \quad$ and $\quad b(f)=-0.099 f_{1}^{4}+b_{0 h}$.

In order to estimate the moment of inertia, one needs to know the minimum value of the compactness parameter $M / R_{\mathrm{eq}}$ for a given spin frequency (right panel in Fig. 8). It can be described by

$\left(M / R_{\mathrm{eq}}\right)_{\min }=a_{\mathrm{c}} f_{1}^{2}+b_{\mathrm{c}}$,

where $b_{\mathrm{c}}=0.005$, and $a_{\mathrm{c}}$ depends on the type of the EOS. For EOSs without hyperons $a_{\mathrm{c}}=0.074$, otherwise $a_{\mathrm{c}}=0.099$. Thanks to their stiffness, hyperonic EOSs produce larger radii near the mass-shedding limit, but the minimum mass for a given $f$ is also higher in comparison to nucleonic EOSs (see Fig. 2), which results in higher $\left(M / R_{\mathrm{eq}}\right)_{\min }$.

\footnotetext{
3 Although $I$ grows with rotation, the value of $I / R^{6}$ decreases because of the high power of $R$ in the denominator. The static results are therefore upper limits for the value of $I / R^{6}$ for a given $M$.
}
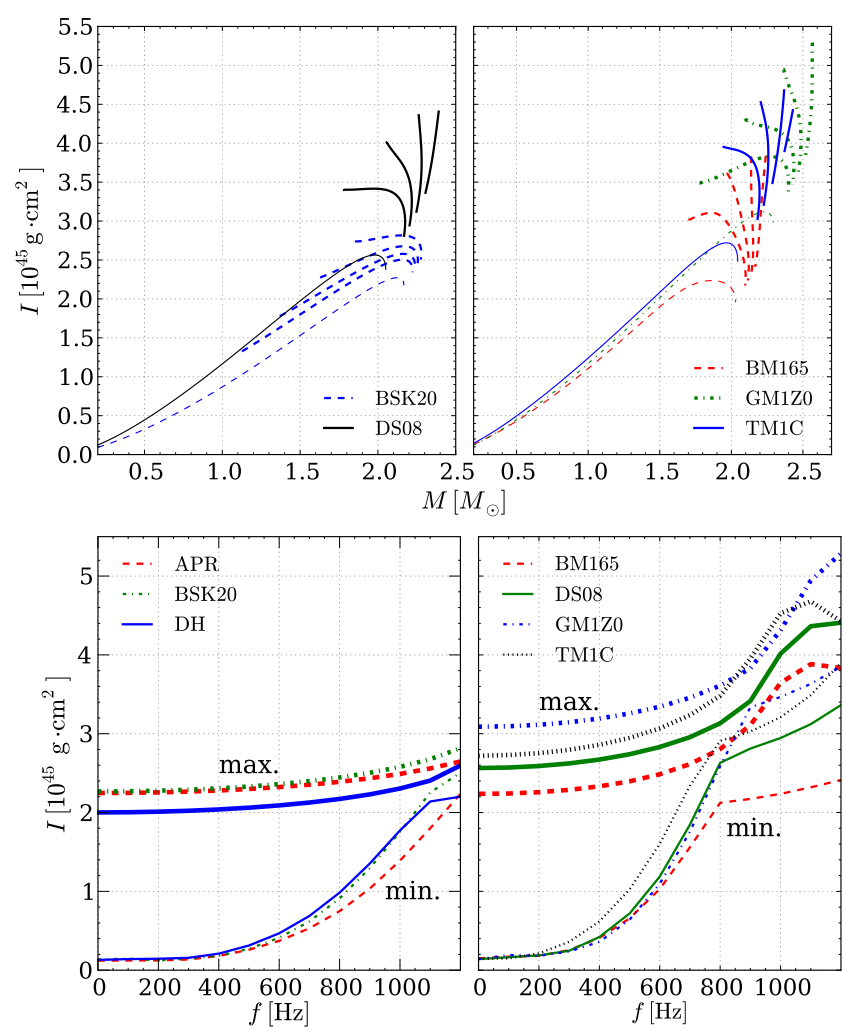

Fig. 7. Top panels: the behavior of $I(M)$ for rotating stars (from left to right $f$ equals $0,900,1000,1100$ and $1200 \mathrm{~Hz}$ ) for hyperonic EOSs and DH EOS for comparison. Bottom panels: allowable range of the moment of inertia for purely nucleonic stars (left) and hyperonic stars (right). Thin (thick) lines correspond to the minimum (maximum) of $I$ that a stable configuration can attain at a given frequency. (Color online).

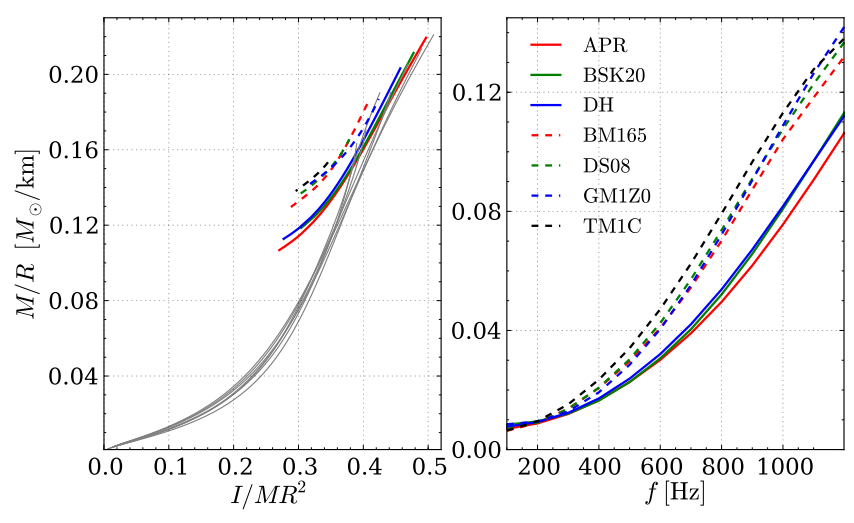

Fig. 8. Left panel: relation between $I / M R^{2}$ and the compactness parameter $M / R$ for static configurations (grey) and those rotating at $1200 \mathrm{~Hz}$, in which case $R \equiv R_{\text {eq }}$. Right panel: minimal $M / R_{\text {eq }}$ ratio (value corresponding to the mass-shedding configuration) as a function of rotational frequency. (Color online.)

\subsection{Spin-up by disk accretion}

A process in which the moment of inertia is relevant is the so-called recycling of pulsars to millisecond periods. To study the relation of spin-up to the EOS we use the model described in Bejger et al. (2011a), a magnetic torque in the form given by Kluźniak \& Rappaport (2007), updated to include the marginally-stable orbit and the magnetic field decay 


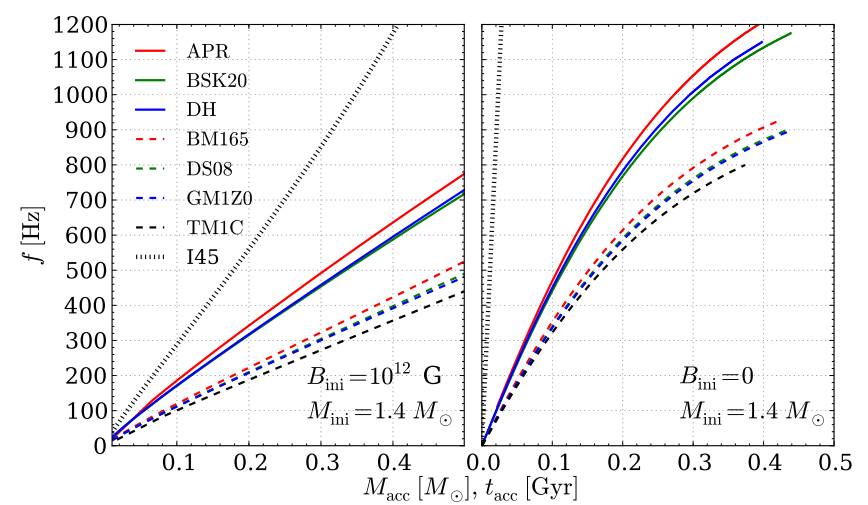

Fig. 9. Spin evolution of an accreting star as a function of the accreted mass (accretion rate is $\dot{M}=10^{-9} M_{\odot} /$ yr, the horizontal axes correspond to accretion time $t_{\text {acc }}$ in Gyr) with initial mass $M_{\text {ini }}=1.4 M_{\odot}$. Left panel: accretion with the magnetic field decay and $B_{\text {ini }}=10^{12} \mathrm{G}$. Right panel: accretion without $B=0$ (from the marginally-stable orbit). (Color online.)

proportional to the amount of accreted mass. The evolution of the total stellar angular momentum in the process of accretion is

$\mathrm{d} J / \mathrm{d} M_{b}=l-l_{\text {mag }}=l_{\text {tot }}$,

where $l$ is the orbital angular momentum of a particle in the disk per unit baryon mass, and $l_{\text {mag }}$ is the contribution from the braking magnetic torque, resulting from the magnetosphere-disk interaction.

For comparison with the realistic EOSs, we employ a useful fiducial model $^{4}$ : I45, a star with a constant radius $R=10 \mathrm{~km}$, and a constant moment of inertia $I=10^{45} \mathrm{~g} \mathrm{~cm}^{2}$. To compare the effect of the EOS on the recycling process, Fig. 9 shows the evolution of spin frequency $f$ for a chosen accretion rate, $\dot{M}=10^{-9} M_{\odot} /$ yr and initial magnetic field $B=10^{12} \mathrm{G}$ (left panel). As anticipated, stars with larger moments of inertia gain $f$ less rapidly. With the above parameters, a hyperonic star has to accrete almost twice as much matter as a nucleonic EOS star to reach given $f$ (see also Bejger et al. 2011a). For comparison, the right panel shows the importance of the magnetic torque. When it is neglected $(B=0)$, the amount of mass needed to spin up the star to a given $f$ is much smaller (lines end at the mass-shedding limit). By rewriting Eq. (7) for $J=I \Omega$,

$\mathrm{d} \Omega / \mathrm{d} M_{b}=\left(l_{\mathrm{tot}}-\Omega \mathrm{d} I / \mathrm{d} M_{b}\right) / I$,

one deduces that both large $I$ and its growth with $M$ decrease the spin-up rate (to be compared with the fiducial I45 configuration; see also the top-left panel in Fig. 6 where we compare the BSK20 and DS08 EOSs). We also note that, contrary to intuition, more massive stars are spinning up faster, as shown in Fig. 10 for $M_{\text {ini }}=1.2 M_{\odot}$ and $M_{\text {ini }}=1.9 M_{\odot}$. This feature results from the fact that $l_{\text {tot }}$ is larger for larger $M$. In the general case, the expression for $l_{\text {tot }}(M)$ is rather complicated (see Bejger et al. 2011a for details); however, for $B=0$ in the Schwarzschild

\footnotetext{
4 In general, the changes of stellar parameters relate to each other as $\mathrm{d} M=\Omega \mathrm{d} J+\mu \mathrm{d} M_{b}$, where $\mu=1 / u^{t}$ is the chemical potential per unit mass, transfered onto the star by the accreting particle, $u^{t}$ being the time component of the particle four-velocity. In the case of $B=0, \mu=$ $e-\Omega l$, where $e$ is the specific energy of a particle infalling onto the star (Sibgatullin \& Sunyaev 2000; Zdunik et al. 2002), the gravitational mass growth of such a star is therefore $\mathrm{d} M=e \mathrm{~d} M_{b}$; we adopt this prescription for $B \neq 0$ too (we also correct a misprint in Eqs. (4) and (5) of Bejger et al. 2011a: instead of $u^{t}$, it should read $1 / u^{t}$ ).
}

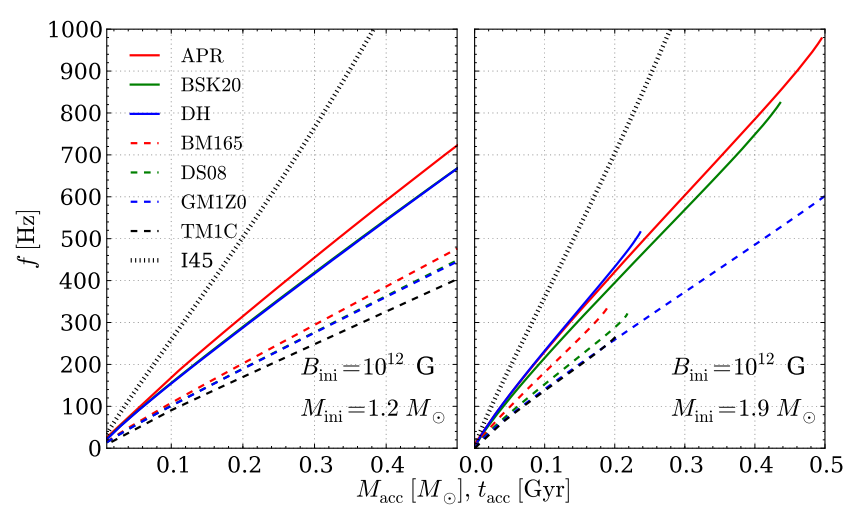

Fig. 10. As in the left panel of Fig. 9, but for $M_{\mathrm{ini}}=1.2 M_{\odot}$ (left panel) and $M_{\mathrm{ini}}=1.9 M_{\odot}$ (right panel). (Color online.)

case, $l \propto \sqrt{M r_{\mathrm{ms}}}$, and $r_{\mathrm{ms}}$, the radius of the innermost stable circular orbit, depends linearly on $M$. This effect is independent of the change of $I$, as shown for the $I=$ const. tracks with different $M_{\text {ini }}$. The change of $\mathrm{d} I / \mathrm{d} M_{b}$ for realistic EOSs, related to the $I(M)$ behavior near the $M_{\max }$, is visible as a slight change of slope in the right panel of Fig. 10, just before the accretion ends at the instability limit.

\subsection{Comparison with stiff nucleonic EOS}

To be consistent with the $2 M_{\odot}$ NS mass measurement, the hyperonic EOSs are necessarily stiff for densities lower than the hyperon appearance threshold. This requirement is not essential for nucleonic EOSs, and is directly reflected in higher compactnesses, smaller radii and smaller moments of inertia of associated stellar models. The nucleonic EOS may however be stiff; to investigate the imprint of hyperons, we construct a purely nucleonic BMN EOS by suppressing hyperons in the BM165 EOS (see Bednarek et al. 2011 for details, where the BMN EOS is called EOS.N). Compared to the BM165 EOS, it yields a moderately higher non-rotating maximum mass, $M_{\max }^{\mathrm{s}}=2.11 M_{\odot}$ and larger $R\left(M_{\mathrm{max}}^{\mathrm{s}}\right), 11.96 \mathrm{~km}$. The lack of hyperons results in available central density ranges that are shifted towards lower values for a given spin frequency, as well as higher moments of inertia (see Fig. 11, in which the left and right panels correspond to Figs. 1 and 7).

Because of the considerable freedom in choosing EOS parameters, the discrimination between a given hyperonic EOS and another stiff, but purely nucleonic EOS is notably hindered, mostly because they give similar values of the stellar radius that affects potentially-measurable parameters. For relations presented in Figs. 1-10, the BMN EOS results resemble those of the DS08 EOS (e.g., in Fig. 5 the central value of $\left(M / R_{\mathrm{eq}}\right)_{\max }$ equals 0.177$)$.

One can thus, in principle, construct a nucleonic EOS that will result in stellar parameters similar to hyperonic models; the reverse statement is, at least up to the current state of the art, not true.

\section{Summary and conclusions}

We have studied a set of recent EOSs that contain hyperons and yield static NSs consistent with a robust observational constraint, a $2 M_{\odot}$ NS mass measured by Demorest et al. (2010). Stars constructed using hyperonic EOSs were compared with those using a minimalistic approach, i.e., containing nucleons only. Their 


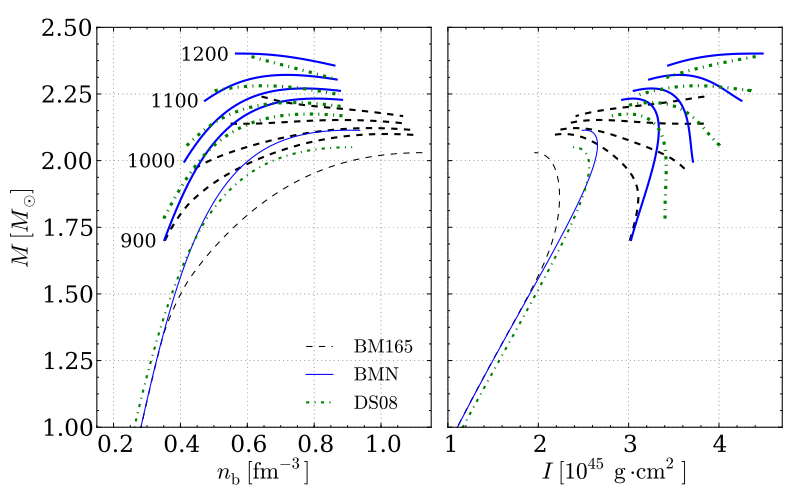

Fig. 11. Gravitational mass-central baryon density $M-n_{\mathrm{b}}$ and the moment of inertia-mass $I(M)$ relations for BM165 (dashed black), BMN (solid blue), and DS08 EOS (dash-dotted green lines). (Color online.)

global parameters, gravitational mass, compactness, surface redshift, and moments of inertia were calculated for a broad range of spin frequencies $(0-1200 \mathrm{~Hz})$. We have focused on the extreme values of these parameters and find that the minimum mass for hyperonic EOSs increases much faster with rotation than in the case of the representative set of nucleonic EOSs described in Sect. 2. For frequencies just slightly larger than $716 \mathrm{~Hz}$ the minimum mass approaches $1.4 M_{\odot}$. In the case of the compactness parameter $M / R_{\text {eq }}$, its minimal value is substantially larger than for nucleonic EOSs at a given $f$ (Fig. 8). We also note that the sub-millisecond rotation confines the mass into a narrow interval (a feature that is present for softer EOS stars for much higher frequencies, see Bejger et al. 2007). However, as shown in Sect. 3.5, stellar models based on a sufficiently stiff nucleonic EOS may be confused with hyperonic EOS models; in view of the measurement of Demorest et al. (2010), an observation of a compact NS (i.e., suggesting a softer nucleonic EOS) with a mass far below $2 M_{\odot}$ would make the existence of hyperons at higher densities less plausible.

A number of approximate formulæ describing the whole set of EOSs is provided. We approximate the relations between the $M_{\max }, M_{\min },\left(M / R_{\mathrm{eq}}\right)_{\min }$, and functions of surface redshift for minimal and maximal compactness configurations for a range of studied frequencies. We find that the maximal compactness configuration has $M / R_{\text {eq }}$ that changes little with rotation.

Because of their stiffness, hyperonic stars have comparatively large moments of inertia that increase with rotation to values significantly larger than the usual $10^{45} \mathrm{~g} \mathrm{~cm}^{2}$. We extend the parametrization of Bejger \& Haensel 2002 by supplying an approximate $I /\left(M R^{2}\right)$ vs. $M / R$ relation for rotating stars. A large moment of inertia is also one of the parameters in the process of recycling a millisecond pulsar that hinders the spin-up. If hyperons exist in the interior of NSs (i.e., the nucleonic matter is sufficiently stiff), it may be one of the reasons high frequencies are not observed.

Acknowledgements. I thank N. Chamel, D. Chatterjee, V. Dexheimer, and M. E. Gusakov for providing their tabulated EOSs, and P. Haensel, J. L. Zdunik, and an anonymous referee for comments. This work was partially supported by the Polish MNiSW research grants No. 2011/01/B/ST9/04838, N N203 512838 and completed using free and open software (LORENE, gnuplot, matplotlib, numpy and scipy).

\section{References}

Akmal, A., Pandharipande, V. R., \& Ravenhall, D. G. 1998, Phys. Rev. C, 58, 1804

Bednarek, I. Haensel, P., Zdunik, J. L., et al. 2012, A\&A, 543, A157

Bejger, M., \& Haensel, P. 2002, A\&A, 396, 917

Bejger, M., Haensel, P., \& Zdunik, J. L. 2007, A\&A, 464, L49

Bejger, M., Zdunik, J. L., \& Haensel, P. 2010, A\&A, 520, A16

Bejger, M., Fortin, M., Haensel, P., \& Zdunik, J. L. 2011a A\&A, 536, A87

Bejger, M., Haensel, P., Zdunik, J. L., \& Fortin, M. 2011b A\&A, 536, A92

Bonazzola, S., \& Gourgoulhon, E. 1994, CQG, 11, 1775

Bonazzola, S., Gourgoulhon, E., Salgado, M., \& Marck, J. A. 1993, A\&A, 278,421

Burgio, G. F., Schulze, H.-J., \& Li, A. 2011, Phys. Rev. C, 83, 025804

Demorest P. B., Pennucci, T., Ransom, S. M., et al. 2010, Nature, 467, 1081

Dexheimer, V., \& Schramm, S. 2008, ApJ, 683, 943

Douchin, F., \& Haensel, P. 2001, A\&A, 380, 151

Friedman, J. L., Ipser, J. R., \& Sorkin, R. D. 1988, ApJ, 325, 722

Goriely, S., Chamel, N., \& Pearson, J. M. 2010, Phys. Rev. C, 82, 035804

Gourgoulhon, E. 2010 [arXiv: 1003. 5015]

Haensel, P., Potekhin, A. Y., \& Yakovlev, D. G. 2007, Neutron Stars 1: Equation of State and Structure (New York: Springer)

Hartle, J. B. 1967, ApJ, 150, 1005

Hessels, J. W. T., Ransom, S. M., Stairs, I. H., et al. 2006, Science, 311, 1901

Kluźniak, W., \& Rappaport, S. 2007, ApJ, 671, 1990

Lattimer, J. M., \& Prakash, M. 2001, ApJ, 550, 426

Lattimer, J. M., \& Schutz, B. F. 2005, ApJ, 629, 979

Schulze, H.-J., Polls, A., Ramos, A., \& Vidaña, I. 2006, Phys. Rev. C, 73, 058801

Sibgatullin, N. R., \& Sunyaev, R. A. 2000, Astron. Lett., 26, 772

Steiner, A. W., Lattimer, J. M., \& Brown, E. F. 2010, ApJ, 722, 33

Vidaña, I., Logoteta, D., Providência, C., et al. 2011, EPL, 94, 11002

Weissenborn, S., Chatterjee, D., \& Schaffner-Bielich, J. 2012, Phys. Rev. C, 85, 065802

Zdunik, J. L., Haensel, P., \& Gourgoulhon, E. 2002, A\&A, 381, 933 